\title{
Implementation of Fourier Expansion Based Differential Quadrature Method (FDQM) and Polynomial Based Differential Quadrature Method (PDQM) for the 2D Helmholtz Problem
}

\author{
Ahmet Rifat GORGUN, Sukru OZEN and Selcuk HELHEL \\ Electrical Electronics Engineering Department, Engineering Faculty, Akdeniz University, 07058 Antalya, Turkey.
}

Accepted 30 August, 2013

\begin{abstract}
The polynomial-based differential quadrature method (PDQM) and Fourier expansion-based differential quadrature method (FDQM) are examined to solve 2D Helmholtz problem expressing definition of many electromagnetic problems. PDQM and FDQM solution of the problems were calculated and compared, analytically. While grid node and wave number are steady, the PDQM and FDQM were applied to 2D Helmholtz problem for calculation and comparison. While the grid node is equal to $7(N=M=7)$ and the wave number is equal to $1(\mathrm{k}=1), \Delta \phi_{\max }$ (maximum absolute error between the numerical result and the exact solution) for PDQM and $\Delta \phi_{\max }$ for FDQM has been found as $2.758 \times 10^{-5}$ and $2.921 \times 10^{-6}$ respectively. Then wave number was increased in order to examine the effects of its variation to the accuracy, while grid node was kept in steady state. While grid node was steady and equal to 7 and the wave number was increased from 1 to $5, \Delta \phi_{\max }$ for FDQM has been found as $2.921 \times 10^{-6}, 7.995 \times 10^{-5}, 1.020 \times 10^{-4}, 0.1825$ and 1.779 respectively. It has been found that the FDQM is more suitable than PDQM for 2D Helmholtz problem having harmonics, and it has been observed that the wave number increases, the accuracy of FDQM results gradually decreases in the case of fixed mesh size and computational domain.
\end{abstract}

Key words: Differential quadrature method (DQM), polynomial-based differential quadrature method (PDQM), Fourier expansion-based differential quadrature method (FDQM), Helmholtz equation.

\section{INTRODUCTION}

Helmholtz equation is the governing equation for many engineering problems such as waveguides in electromagnetic fields, vibration of membranes and water wave diffraction in offshore structure engineering (Shu and Chew, 1999). In addition, acoustic waves and microwaves can be simulated by the Helmholtz equation. The numerical solutions of the 2D Helmholtz problems can be obtained by several methods such as finite difference method and finite element method. FDM and
FEM fall under the category of low order methods. The FDM is based on the Taylor series expansion while the FEM is based on the principle weighted residuals. Most numerical solutions of engineering problems can be obtained by the low order FDM instead of FEM which is using large number of grid points. In some applications, however, the numerical solutions of partial differential equation are required at only a few specified points in the physical domain. To achieve an acceptable degree of 
accuracy, FEM and FDM still require the use of a large number of grid points.

A partial derivative of a function with respect to coordinate direction is expressed as a linear weighted sum of all the functional values at all mesh points along the direction. DQM is an efficient approach to solve the 2D Helmholtz problem. There are two versions of DQM. One is noted as polynomial-based differential quadrature (PDQ) approach. The solution of PDE is approximated by a polynomial of high degree in PDQ approach. Civan and Sliepcevich $(1983,1984)$ extended and generalized the method of $D Q$ and applied it to the Poisson equation and multidimensional problems. The FDQ approach was developed by Shu and Chew (1997) and Shu and Xue (1999). Shu, Chew and Xue have further developed some simple algebraic formulations to compute, such as the weighting coefficients of the first and second order derivatives in the DQ approach when the function or the solution of a PDE is approximated by a Fourier series expansion. Qinwei and Mazumder (2002) adapted DQM to model lossy uniform and non uniform transmission lines. Tang et al. (2005) applied DQM to analyse interconnects with frequency-dependent parameters. Tang and Mao (2008) applied the differential quadrature method (DQM) with Chebyshev-Gauss-Lobatto (CGL) sampling points for transient analysis of multiconductor transmission lines. Previous application of FDQM and PDQM showed that accurate numerical solutions can be obtained by using a considerably small number of grid points. In this study, PDQM and FDQM are applied to solve the 2D Helmholtz problem. The accuracy of PDQM and FDQM are validated by their application to the $2 D$ Helmholtz problem, which have exact solution.

\section{DIFFERENTIAL QUADRATURE METHOD (DQM)}

DQM is a numerical discrimination technique for the approximation of derivatives. The integral $\int_{a}^{b} f(x) d x$ can be approximated by

$$
\int_{a}^{b} f(x) d x=w_{1} f_{1}+w_{2} f_{2}+\ldots . .+w_{n} f_{n}=\sum_{k=1}^{n} w_{k} f_{k}
$$

Where $w_{1}, w_{2}, \ldots, w_{n}$ are the weighting coefficients, $f_{1}, f_{2}, \ldots, f_{n}$ are the functional values at the discrete points. It is called the integral quadrature and integral quadrature uses all the functional values in the whole integral domain to approximate an integral over a finite interval.

The method of differential quadrature approximates the partial derivative of a function with respect to a space variable at a given discrete point as weighted linear sum of function values at all the discrete points in the domain of that variable. The first and second order derivatives of $f(x)$ at a point $x_{i}$ are approximated by

Gorgun et al.

1671

$$
\begin{aligned}
& f_{x}\left(x_{i}\right)=\left.\frac{d f}{d x}\right|_{x_{i}}=\sum_{j=1}^{N} a_{i j} \cdot f\left(x_{j}\right) \\
& f_{x x}\left(x_{i}\right)=\left.\frac{d^{2} f}{d x^{2}}\right|_{x_{i}}=\sum_{j=1}^{N} b_{i j} \cdot f\left(x_{j}\right)
\end{aligned}
$$

Where, $i=1,2,3, \ldots . N, a_{i j}$ and $b_{i j}$ represent the weighting coefficient, $f\left(x_{j}\right)$ represents the functional value at a grid point $x_{j}, \mathrm{~N}$ is the number of grid points in the whole domain. Equations 2 and 3 are called DQ. 2 and 3 are similar equations but they use different weighting coefficients. The key procedure in DQ approximation is to determine the weighting coefficients.

\section{POLYNOMIAL BASED DIFFERENTIAL QUADRATURE METHOD (PDQM)}

PDQM determine the weighting coefficients in the $D Q$ approximation when the solution of the PDE is approximated by a polynomial of high degree. Weighting coefficients can be efficiently computed by some explicit formulations. One of the approaches to determine the weighting coefficients of first and second order derivatives is Quan and Chang (1989a, b) approach. Quan and Chang use the Lagrange interpolation polynomials and then derived

$$
\begin{aligned}
& a_{i j}=\frac{1}{x_{j}-x_{i}} \prod_{k=1, k \neq j}^{N} \frac{x_{i}-x_{k}}{x_{j}-x_{k}}, \text { for } j \neq i \\
& a_{i i}=\sum_{k=1, k \neq i}^{N} \frac{1}{x_{i}-x_{k}} \\
& b_{i j}=\frac{2}{x_{j}-x_{i}}\left(\prod_{k=1, k \neq i}^{N} \frac{x_{i}-x_{k}}{x_{j}-x_{k}}\right)\left(\sum_{l=1, l \neq i}^{N} \frac{1}{x_{i}-x_{l}}\right), \text { for } i \neq j
\end{aligned}
$$

$$
b_{i i}=2 \sum_{k=1, k \neq i}^{N-1}\left[\frac{1}{x_{i}-x_{k}}\left(\sum_{l=k, l \neq i}^{N} \frac{1}{x_{i}-x_{l}}\right)\right]
$$

\section{FOURIER EXPANSION BASED DIFFERENTIAL QUADRATURE METHOD (FDQM)}

A way of computation of weighting function is the difference between PDQM and FDQM. It is supposed that $f(x)$ is approximated by a Fourier series expansion of the form given in Equation 8.

$$
f(x)=c_{0}+\sum_{k=1}^{N / 2}\left(c_{k} \cos k x+d_{k} \sin k x\right)
$$

It is easy to show that $f(x)$ in Equation 8 constitutes a $(\mathrm{N}+1)$ dimensional linear vector space $V_{N+1}$. Two typical sets of base vectors in $V_{N+1}$ are

1672 Sci. Res. Essays 
$V_{N+1}=1, \cos x, \sin x, \cos 2 x, \sin 2 x, \ldots, \sin (N x / 2)$

$$
s_{k}(x)=\frac{S(x)}{q\left(x_{k}\right) \times \sin \left(\frac{x-x_{k}}{2}\right)} \quad \mathrm{k}=0,1,2, \ldots, \mathrm{N}
$$

$\mathrm{S}(\mathrm{x})=\prod_{k=0}^{N} \sin \frac{x-x_{k}}{2}$

$$
q\left(x_{i}\right)=\prod_{k=0, k \neq i}^{N} \sin \frac{x_{i}-x_{k}}{2}
$$

We use the above two sets of base vectors to derive explicit formulations to compute the weighting coefficients of the first, second and third order derivatives.

$$
\begin{aligned}
& \sum_{j=0}^{N} a_{i j}=0 \text { or } a_{i i}=-\sum_{j=0, j \neq i}^{N} a_{i j} \\
& \sum_{j=0}^{N} b_{i j}=0 \text { or } b_{i i}=-\sum_{j=0, j \neq i}^{N} b_{i j}
\end{aligned}
$$

Equations $12 \mathrm{a}$ and $12 \mathrm{~b}$ are used to compute the diagonal weighting coefficients. Finally, we obtain:

$$
\begin{aligned}
& a_{i j}=\frac{q\left(x_{i}\right)}{2 \cdot \sin \frac{x_{i}-x_{j}}{2} \cdot q\left(x_{j}\right)}, j \neq i \\
& b_{i j}=a_{i j}\left[2 a_{i i}-\cot \frac{x_{i}-x_{j}}{2}\right], \quad j \neq i
\end{aligned}
$$

The two dimensional Helmholtz equation can then be written as:

$$
\frac{\partial^{2} \phi}{\partial x^{2}}+\frac{\partial^{2} \phi}{\partial y^{2}}+k^{2} \phi=f(x, y)
$$

Application of the DQM to discrete the derivative in Helmholtz Equation leads to:

$$
\sum_{k=1}^{N} W_{i k}^{(2)} \phi_{k j}+\sum_{k=1}^{M}{\overline{W_{j k}}}^{(2)} \phi_{i k}+k^{2} \phi_{i j}=f_{i j}
$$

Where $\mathrm{N}$ and $\mathrm{M}$ are the number of grid points in the $\mathrm{x}$ and $\mathrm{y}$ directions respectively, $W_{i k}^{(2)}$ and $\overline{W_{j k}^{(2)}}$ are the weighting coefficients of the second order derivatives with respect to $\mathrm{x}$ and $\mathrm{y}$. $W_{i k}^{(2)}$ and $\overline{W_{j k}^{(2)}}$ are computed by Equations 6 and 7 for PDQM, while $W_{i k}^{(2)}$ and $\overline{W_{j k}^{(2)}}$ are computed by Equations $11,12 \mathrm{a}, 12 \mathrm{~b}$, 13 and 14 for the FDQ approach. In this study, both PDQM and FDQM use the

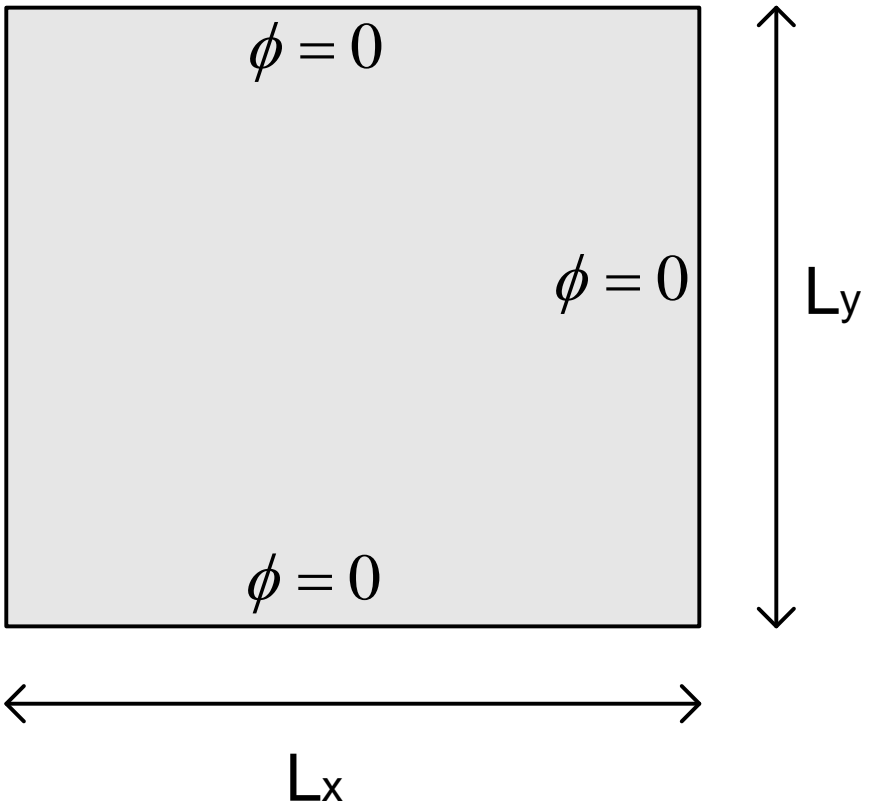

Figure 1. 2D Helmholtz problem.

following Chebyshev-Gauss-Lobatto (Shu and Richards, 1992) point distribution as in Equations 17 and 18. $0 \leq X \leq L_{x}$, $0 \leq Y \leq L_{y}$

$X_{i}=\frac{1}{2}\left[1-\cos \left(\frac{i-1}{N-1} \pi\right)\right] L_{x}, \mathrm{i}=1,2, \ldots, \mathrm{N}$

$Y_{j}=\frac{1}{2}\left[1-\cos \left(\frac{j-1}{M-1} . \pi\right)\right] \times L_{y}, \mathrm{j}=1,2, \ldots, \mathrm{M}$

$f(x, y)$ is supposed to be expressed as in Equation 19

$f(x, y)=k^{2}\left[1-\pi^{2}\left(\frac{1}{L_{x}^{2}}+\frac{1}{L_{y}^{2}}\right] \sin \frac{k \pi x}{L_{x}} \sin \frac{k \pi y}{L_{y}}\right.$

When the Dirichlet boundary condition of $\phi=0$ is imposed on the boundary as seen in Figure 1, the problem has an exact solution as $\phi=\sin \frac{k \pi x}{L_{x}} \sin \frac{k \pi y}{L_{y}} . \mathrm{L}_{\mathrm{x}}$ and $\mathrm{L}_{\mathrm{y}}$ are taken as $\pi$, the number of grid points used in the $x$ and $y$ directions is taken the same and is noted N, M, respectively. The accuracy of numerical results for this problem is measured by $\Delta \phi$. $\Delta \phi=\left|\phi_{i j}-\phi_{\text {exact }}\left(x_{i}, y_{j}\right)\right|$ where $\phi_{i j}$ is the numerical solution at the mesh points $\left(x_{i}, y_{j}\right)$, and $\phi_{\text {exact }}\left(x_{i}, y_{j}\right)$ is the exact solution at the same mesh point. $\phi$ and $\mathrm{k}$ are respectively the potential and the wave number defined in the two dimensional domain. Weighting coefficients of the first and second order derivatives are computed for the PDQM and FDQM. 
Table 1. $\Delta \phi$ of $\mathrm{PDQM}$ for $\mathrm{N}=\mathrm{M}=5$.

\begin{tabular}{ccccccc}
\hline & & $\mathbf{X}_{1}$ & $\mathbf{X}_{2}$ & $\mathbf{X}_{3}$ & $\mathbf{X}_{4}$ & $\mathbf{X}_{5}$ \\
\cline { 3 - 7 } & 0 & 0.46 & 1.57 & 2.68 & 3.14 \\
$\mathbf{Y}_{1}$ & 0 & 0 & 0 & 0 & 0 & 0 \\
$\mathbf{Y}_{2}$ & 0.46 & 0 & 0.0012 & 0.0036 & 0.0012 & 0 \\
$\mathbf{Y}_{3}$ & 1.57 & 0 & 0.0036 & 0.0102 & 0.0036 & 0 \\
$\mathbf{Y}_{4}$ & 2.68 & 0 & 0.0012 & 0.0036 & 0.0012 & 0 \\
$\mathbf{Y}_{5}$ & 3.14 & 0 & 0 & 0 & 0 & 0 \\
\hline
\end{tabular}

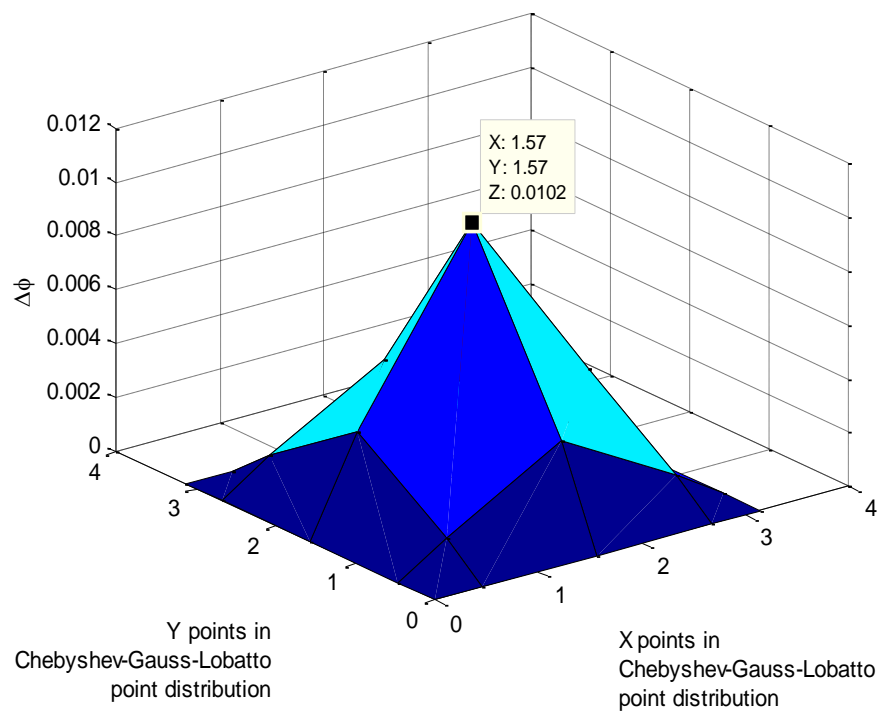

Figure 2. $\Delta \phi$ of $\mathrm{PDQM}$ for $\mathrm{N}=\mathrm{M}=5$.

\section{RESULTS AND DISCUSSION}

Proposed problem suggests Lx and Ly are $\pi$, and number of grid points used in the $x$ and $y$ directions are the same. Finally, the function $f(x, y)$ can be written as in Equation 20.

$$
f(x, y)=k^{2} \times\left[1-\pi^{2}\left(\frac{1}{L_{x}^{2}}+\frac{1}{L_{y}^{2}}\right)\right] \sin \frac{k \pi x}{L_{x}} \sin \frac{k \pi y}{L_{y}}
$$

When the Dirichlet boundary condition of $\phi=0$ is imposed on the boundary, the problem has an exact solution as in Equation 21.

$\phi=\sin \frac{k \pi x}{L_{x}} \sin \frac{k \pi y}{L_{y}}$

Table 1 contains $X_{i}$ and $Y_{j}$ which were obtained by Chebyshev-Gauss-Lobatto point distribution for $L_{x}=L_{y}=\pi$ computational domain in the case of

Gorgun et al. $\quad 1673$
Table 2. $\Delta \phi$ of FDQM for $\mathrm{N}=\mathrm{M}=5$ ( $\mathrm{x} 1.0 \mathrm{e}-004)$.

\begin{tabular}{ccccccc}
\hline & & $\mathbf{X}_{1}$ & $\mathbf{X}_{2}$ & $\mathbf{X}_{3}$ & $\mathbf{X}_{4}$ & $\mathbf{X}_{5}$ \\
\cline { 3 - 7 } & & 0 & 0.46 & 1.57 & 2.68 & 3.14 \\
$\mathbf{Y}_{1}$ & 0 & 0 & 0 & 0 & 0 & 0 \\
$\mathbf{Y}_{2}$ & 0.46 & 0 & 0.0616 & 0.1087 & 0.0543 & 0 \\
$\mathbf{Y}_{3}$ & 1.57 & 0 & 0.1087 & 0.1770 & 0.0922 & 0 \\
$\mathbf{Y}_{4}$ & 2.68 & 0 & 0.0543 & 0.0922 & 0.0470 & 0 \\
$\mathbf{Y}_{5}$ & 3.14 & 0 & 0 & 0 & 0 & 0 \\
\hline
\end{tabular}

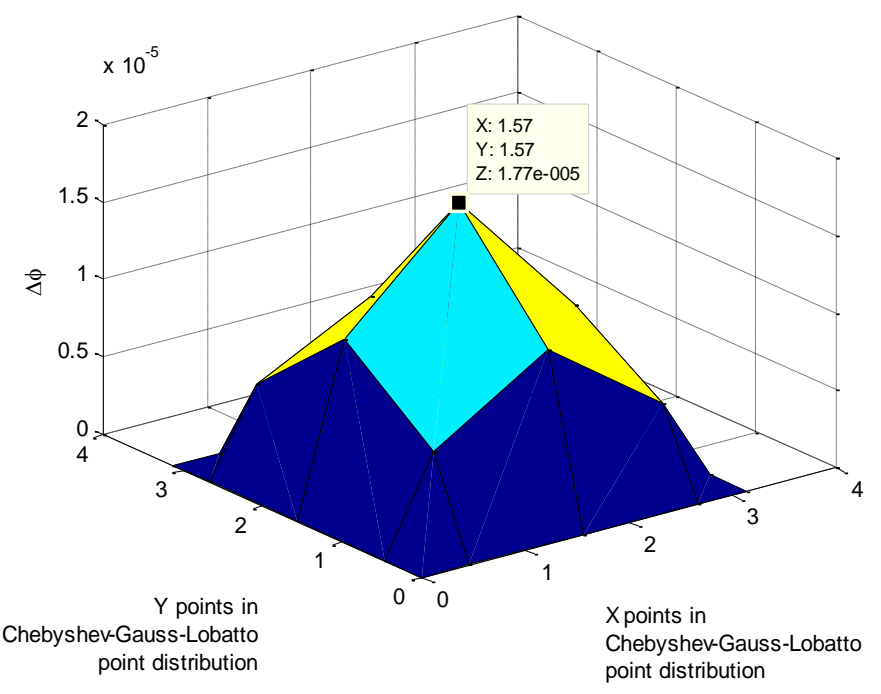

Figure 3. $\Delta \phi$ of $\mathrm{FDQM} \mathrm{N}=\mathrm{M}=5$.

$\mathrm{N}=\mathrm{M}=5$, grid node. Figure 2 shows that maximum error $\left(\Delta \phi_{\max }\right)$ has been obtained as $1.02 \times 10^{-2}$ by using PDQM.

Table 2 contains $X_{i}$ and $Y_{j}$ which were obtained by Chebyshev-Gauss-Lobatto point distribution for $L_{x}=L_{y}=\pi$ computational domain in the case of $\mathrm{N}=\mathrm{M}=5$, grid node. Figure 3 shows that maximum error $\left(\Delta \phi_{\max }\right)$ has been obtained as $1.77 \times 10^{-5}$ by using FDQM.

Table 3 contains $X_{i}$ and $Y_{j}$ which were obtained by Chebyshev-Gauss-Lobatto point distribution for $L_{x}=L_{y}=\pi$ computational domain in the case of $\mathrm{N}=\mathrm{M}=7$, grid node. Figure 4 shows that maximum error $\left(\Delta \phi_{\max }\right)$ has been obtained as $2.758 \times 10^{-5}$ by using PDQM.

Table 4 contains $X_{i}$ and $Y_{j}$ which were obtained by Chebyshev-Gauss-Lobatto point distribution for $L_{x}=L_{y}=\pi$ computational domain in the case of $\mathrm{N}=\mathrm{M}=7$, grid node. Figure 5 shows that maximum error $\left(\Delta \phi_{\max }\right)$ has been obtained as $2.921 \times 10^{-6}$ by using FDQM. 1674 Sci. Res. Essays 


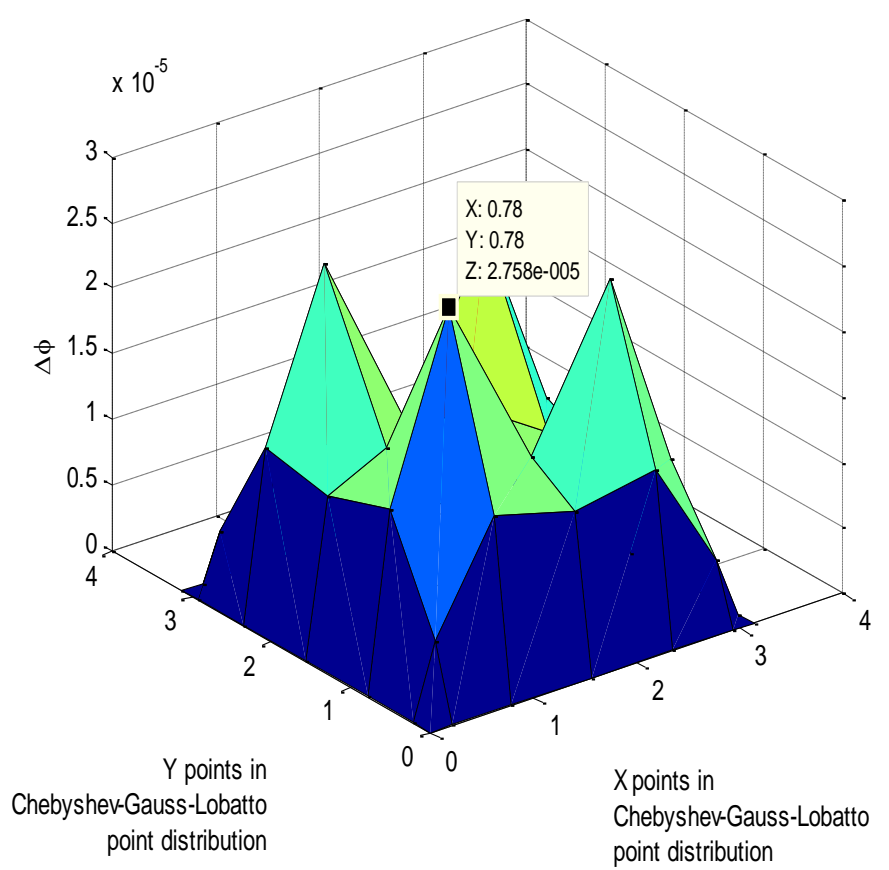

Figure 4. $\Delta \phi$ of $\mathrm{PDQM}$ for $\mathrm{N}=\mathrm{M}=7$.

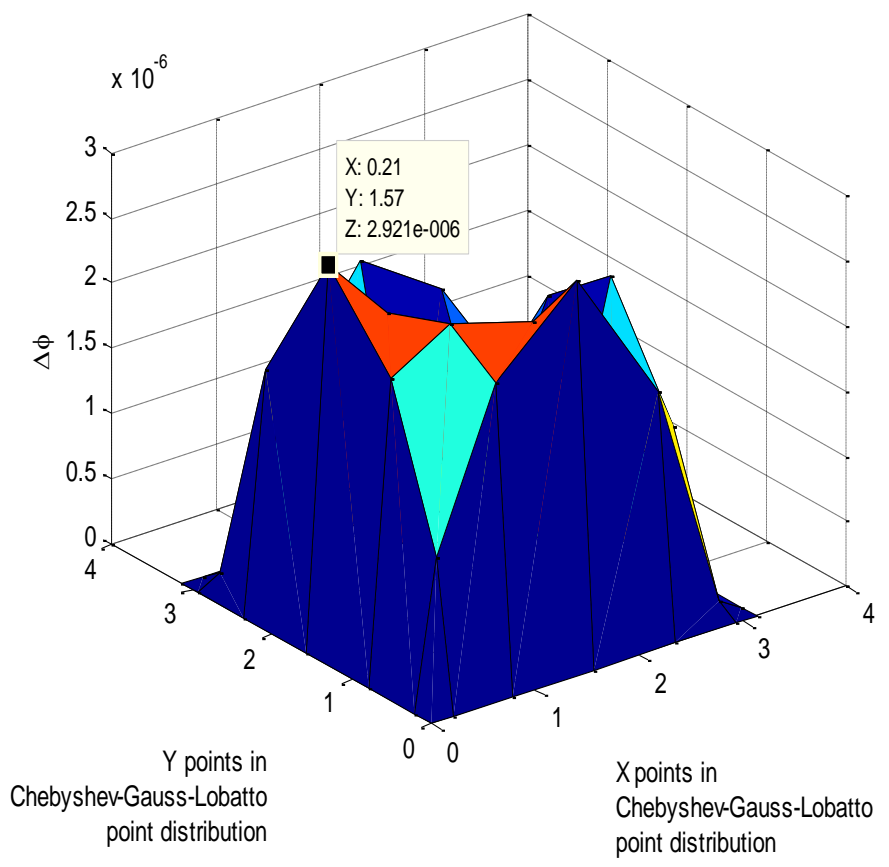

Figure 5. $\Delta \phi$ of FDQM for $\mathrm{N}=\mathrm{M}=7$.

Table 5 brings in understanding that FDQ approach is more suitable than other methods for 2D Helmholtz type problems having harmonic behavior in the case of similar mesh size usage.
Table 6 shows the maximum absolute error $\Delta \phi$ between the numerical results and the exact solution for $\mathrm{k}=1,2,3,4,5$. It can be observed from Table 6 that for a fixed mesh size and computational domain, as the wave number is increased, the accuracy of FDQM results gradually decrease.

This study, the wave number $(k)$, problem solution regions $L_{x}=L_{y}=\pi$, and $\mathrm{xi}$, yi from calculations by Chebyshev-Gauss-Lobatto point distribution, and the results relation to $\Delta \phi$ have been evaluated and given as detailed in Table and graphics. This study has not only focused on $\Delta \phi_{\max }$, the present study includes more numerical details which is valuable for the readers.

\section{CONCLUSIONS}

This study presents the application of PDQM and FDQM to solve 2D Helmholtz problem. As seen in Table 5, while the grid node is equal to $5(\mathrm{~N}=\mathrm{M}=5)$ and the wave number is equal to $1(\mathrm{k}=1), \Delta \phi_{\max }$ for PDQM and $\Delta \phi_{\max }$ for FDQM have been found as $1.02 \times 10^{-2}$ and $1.77 \times 10^{-5}$, respectively. FDQM predicts results which is 576 times better than PDQM for grid nodes of $\mathrm{N}=\mathrm{M}=5$. For the grid node of $\mathrm{N}=\mathrm{M}=7$ and the wave number $\mathrm{k}=1, \Delta \phi_{\max }$ for PDQM and $\Delta \phi_{\max }$ for FDQM have been obtained as $2.758 \times 10^{-5}$ and $2.921 \times 10^{-6}$, respectively. For this state, FDQM predicts results which is about 9 times better then PDQM for grid nodes of $\mathrm{N}=\mathrm{M}=7$.

For the computational domain of $L_{x}=L_{y} \pi$ and the wave number $\mathrm{k}=1$ with $\mathrm{N}=\mathrm{M}=7, \Delta \phi_{\max }$ for $\mathrm{FDQM}$ equals to $2.921 \times 10^{-6}$ as demonstrated in Table 6 . While the wave number $\mathrm{k}$ varies between 1 and 5 , and mesh size and computational domain are fixed, $\Delta \phi_{\max }$ for FDQM has been found as 1.7792. FDQM for $k=5$ predicts 60,000 times better results than FDQM for $k=1$. This study has not only focused on $\Delta \phi_{\max }$; it can be concluded that the FDQM obtains more accurate numerical results than the PDQM. For a fixed mesh size and computational domain, increased wave number results in decreasing accuracy of FDQM. This method can be beneficial for numeric solutions of Helmholtz based problems such as acoustic waves and microwaves.

\section{ACKNOWLEDGEMENT}

This study is supported by Akdeniz University, Scientific Research Projects Supporting Unit (BAPYB). 
Table 3. $\Delta \phi$ of PDQM for $\mathrm{N}=\mathrm{M}=7\left(1.0 \mathrm{e}-004^{\star}\right)$.

\begin{tabular}{ccccccccc}
\hline & & $\mathbf{X}_{1}$ & $\mathbf{X}_{2}$ & $\mathbf{X}_{3}$ & $\mathbf{X}_{4}$ & $\mathbf{X}_{5}$ & $\mathbf{X}_{6}$ & $\mathbf{X}_{\mathbf{7}}$ \\
\cline { 3 - 8 } & & 0 & 0.21 & 0.78 & 1.57 & 2.35 & 2.93 & 3.14 \\
$\mathbf{Y}_{1}$ & 0 & 0 & 0 & 0 & 0 & 0 & 0 & 0 \\
$\mathbf{Y}_{2}$ & 0.21 & 0 & 0.0566 & 0.1366 & 0.1196 & 0.1306 & 0.0468 & 0 \\
$\mathbf{Y}_{3}$ & 0.78 & 0 & 0.1366 & 0.2758 & 0.1411 & 0.2558 & 0.1033 & 0 \\
$\mathbf{Y}_{4}$ & 1.57 & 0 & 0.1196 & 0.1411 & 0.1526 & 0.1128 & 0.0725 & 0 \\
$\mathbf{Y}_{5}$ & 2.35 & 0 & 0.1306 & 0.2558 & 0.1128 & 0.2358 & 0.0974 & 0 \\
$\mathbf{Y}_{6}$ & 2.93 & 0 & 0.0468 & 0.1033 & 0.0725 & 0.0974 & 0.0369 & 0 \\
$\mathbf{Y}_{7}$ & 3.14 & 0 & 0 & 0 & 0 & 0 & 0 & 0 \\
\hline
\end{tabular}

Table 4. $\Delta \phi$ of $\mathrm{FDQM}$ for $\mathrm{N}=\mathrm{M}=7\left(1.0 \mathrm{e}-005^{\star}\right)$.

\begin{tabular}{ccccccccc}
\hline & & $\mathbf{X}_{\mathbf{1}}$ & $\mathbf{X}_{\mathbf{2}}$ & $\mathbf{X}_{\mathbf{3}}$ & $\mathbf{X}_{\mathbf{4}}$ & $\mathbf{X}_{5}$ & $\mathbf{X}_{6}$ & $\mathbf{X}_{\mathbf{7}}$ \\
\cline { 2 - 8 } & 0 & 0.21 & 0.78 & 1.57 & 2.35 & 2.93 & 3.14 \\
$\mathbf{Y}_{\mathbf{1}}$ & 0 & 0 & 0 & 0 & 0 & 0 & 0 & 0 \\
$\mathbf{Y}_{\mathbf{2}}$ & 0.21 & 0 & 0.1151 & 0.2332 & 0.2921 & 0.1856 & 0.0098 & 0 \\
$\mathbf{Y 3}_{3}$ & 0.78 & 0 & 0.2332 & 0.2595 & 0.2397 & 0.0987 & 0.1235 & 0 \\
$\mathbf{Y}_{\mathbf{4}}$ & 1.57 & 0 & 0.2921 & 0.2397 & 0.1591 & 0.0123 & 0.2122 & 0 \\
$\mathbf{Y}_{\mathbf{5}}$ & 2.35 & 0 & 0.1856 & 0.0987 & 0.0123 & 0.0621 & 0.1710 & 0 \\
$\mathbf{Y}_{\mathbf{6}}$ & 2.93 & 0 & 0.0098 & 0.1235 & 0.2122 & 0.1710 & 0.0956 & 0 \\
$\mathbf{Y}_{\mathbf{7}}$ & 3.14 & 0 & 0 & 0 & 0 & 0 & 0 & 0 \\
\hline
\end{tabular}

Table 5. Comparison of $\Delta \phi_{\text {max }}$ for the 2D Helmholtz problem $\left(\Delta \phi_{\max }=\max \left|\phi_{i j}-\phi_{\text {exact }}\left(x_{i}, y_{j}\right)\right|\right)$.

\begin{tabular}{lcc}
\hline Grids & $\mathbf{N}=\mathbf{M}=5$ & $\mathbf{N}=\mathbf{M}=\mathbf{7}$ \\
\hline PDQM $\left(L_{x}=L_{y}=\pi\right.$ and $\left.k=1\right)$ & $1.02 \times 10^{-2}$ & $2.758 \times 10^{-5}$ \\
FDQM $\left(L_{x}=L_{y}=\pi\right.$ and $\left.k=1\right)$ & $1.77 \times 10^{-5}$ & $2.921 \times 10^{-6}$ \\
\hline
\end{tabular}

Table 6. Comparison of $\Delta \phi_{\text {max }}$ for the 2D Helmholtz problem.

\begin{tabular}{lc}
\hline Grids & $\mathbf{N}=\mathbf{M}=7$ \\
\hline $\operatorname{FDQM}\left(L_{x}=L_{y}=\pi\right.$ and $\left.\mathrm{k}=1\right)$ & $2.921 \times 10^{-6}$ \\
$\operatorname{FDQM}\left(L_{x}=L_{y}=\pi\right.$ and $\left.\mathrm{k}=2\right)$ & $7.995 \times 10^{-5}$ \\
$\operatorname{FDQM}\left(L_{x}=L_{y}=\pi\right.$ and k=3) & $1.020 \times 10^{-4}$ \\
$\operatorname{FDQM}\left(L_{x}=L_{y}=\pi\right.$ and k=4) & $1.825 \times 10^{-1}$ \\
$\operatorname{FDQM}\left(L_{x}=L_{y}=\pi\right.$ and k=5) & 1.7792 \\
\hline
\end{tabular}

\section{REFERENCES}

Civan F, Sliepcevich CM (1983). Solution of the Poisson equation by differential quadrature. Int. J. Numer. Meth. Eng. 19:711-724
Civan F, Sliepcevich CM (1984). Differential quadrature for multidimensional problems. J. Math. Anal. Appl. 101:423-443.

Qinwei X, Mazumder P (2002). Accurate modeling of lossy non uniform transmission lines by using differential quadrature method. IEEE Trans. Microw. Theory Tech. 50(10):2233-2246.

Quan JR, Chang CT (1989a). New insights in solving distributed system equations by the quadrature methods. Comp. Chem. Eng. 13:779788.

Quan JR, Chang CT (1989b). New insights in solving distributed system equations by the quadrature methods. Comp. Chem. Eng. 13:10171024.

Shu C, Chew YT (1997). Fourier expansion-based differential quadrature and its application to Helmholtz eigenvalue problem. Com. Numer. Meth. Eng. 13(8):643-653.

Shu C, Chew YT (1999). Application of multi-domain GDQ method to analysis of waveguides with rectangular boundaries. In: Kong Ja Electromagnetic Waves: Pier 21. Emw Publishing, Cambridge, Massachusetts, USA.

Shu C, Richards BE (1992). Application of generalized differential quadrature to solve two dimensional incompressible Navier-Stokes equations. Int. J. Numer. Meth. Fluids 15:791-798.

Shu C, Xue H (1999). Solution of Helmholtz equation by differential quadrature method. Com. Meth. Appl. M. 175(1-2):203-212.

Tang M, Mao JF (2008). A differential quadrature method for the transient analysis of multiconductor transmission lines. Int. Conf. Microw. Millimeter Wave Technol. ICMMT2008 3:1423-1426.

Tang M, Mao JF, Li XC (2005). Analysis of interconnects with frequency-dependent parameters by differential quadrature method. Microw. Wireless Components Lett. IEEE 15(12):877-879. 